\title{
Monochromated Low-Voltage EELS of Optical Resonances in Quantum Materials
}

\author{
David C. Bell ${ }^{1}$, Felix von Cube ${ }^{1}$ and Peter Rez ${ }^{2}$ and Toshihiro Aoki ${ }^{2}$ \\ ${ }^{1}$ Harvard John A. Paulson School of Engineering and Applied Sciences, Harvard University, Cambridge, \\ MA, USA \\ ${ }^{2}$ Arizona State University, Tempe, AZ, USA
}

Depending on the composition, Quantum Materials may act as conductors, insulators, semiconductors or even as superconductors. Combinations of different quantum materials are of high interest to explore new phenomena and act as the foundation for future electronic devices at the nanometer scale. Our quantum materials research is widely spread, reaching from defect formation in graphene to the characterization of hybrid quantum materials. We present our work utilizing Low-Voltage Monochromated EELS and Low-Voltage High-Resolution Electron Microscopy (LV HREM). Together, these often improve the contrast to damage ratio obtained on a large class of samples.

We have imaged and produced EELS spectra of Nitrogen Vacancy (NV) centers in diamond with our Zeiss Libra TEM [1] with a monochromated electron source and in-column energy filter, Fig. 1. With monochromated electron energy loss spectroscopy (EELS) we measure the amount of energy loss that an electron undergoes; this includes optical resonances and inter and intra band transitions but also of course the Cherenkov radiation background. The low acceleration voltage of $40 \mathrm{kV}$ directly reduces the background noise of the Cherenkov radiation. According to the photoluminescence spectra, we find a resonance at $1.9 \mathrm{eV}$ (corresponding to $638 \mathrm{~nm}$ wavelength). We have also demonstrated an unexpectedly strong surface-plasmonic absorption at the interface of silver and high-index dielectrics based on electron and photon spectroscopy [2]. The measured bandwidth and intensity of absorption deviate significantly from the classical theory. Our density-functional calculation well predicts the occurrence of this phenomenon. It reveals that due to the low metal-to-dielectric work function at such interfaces, conduction electrons can display a drastic quantum spillover, causing the interfacial electron-hole pair production to dominate the decay of surface plasmons (Fig. 2). This finding can be of fundamental importance in understanding and designing quantum nanoplasmonic devices that utilize noble metals and high-index dielectrics.

$\mathrm{Fe}_{3} \mathrm{Sn}_{2}$ is a rare metallic Kagome ferromagnet, which synthesis as a single crystal has not previously been reported. We study this single crystal as well as other topological insulators with the particular interest in the correlated behavior in topologically non-trivial materials. The (S)TEM images mapped with low voltage EELS show the atomic structure of the layered material, and the magnetic force microscopy measurements reveal the magnetic anisotropy of the crystal on the surface. Monochromated EELS is the key to all this work, due to increased signal to noise and background and/or ZLP reduction. [3]

References:

[1] D.C. Bell, C.J. Russo and D. Kolmykov, Ultramicroscopy. 114 (2012), p 31.

[2] D. Jin et al, Phys. Rev. Lett., 115(19) (2015), p.193901

[3] This work was supported by the STC, Center for Integrated Quantum Materials, NSF Grant No.

DMR-1231319 

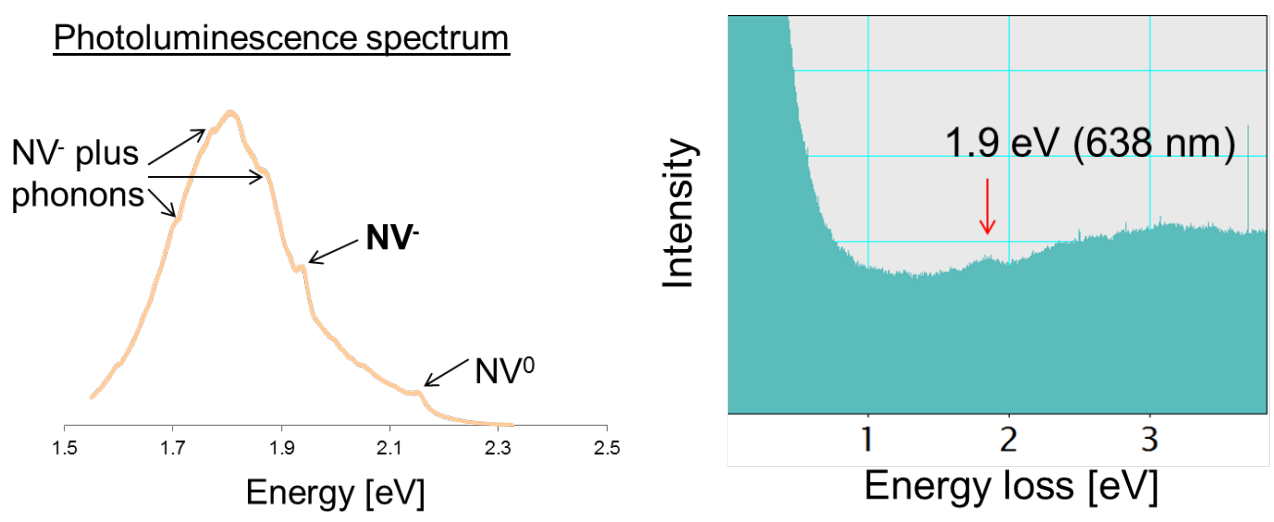

Figure 1. LV-EELS of Nitrogen Vacancy centers in diamond. Left, the corresponding PL spectra of NV centers in a sample of thin diamond. Right, the Low Voltage Monochromated LV-EELS spectra from the same sample showing NV signal.

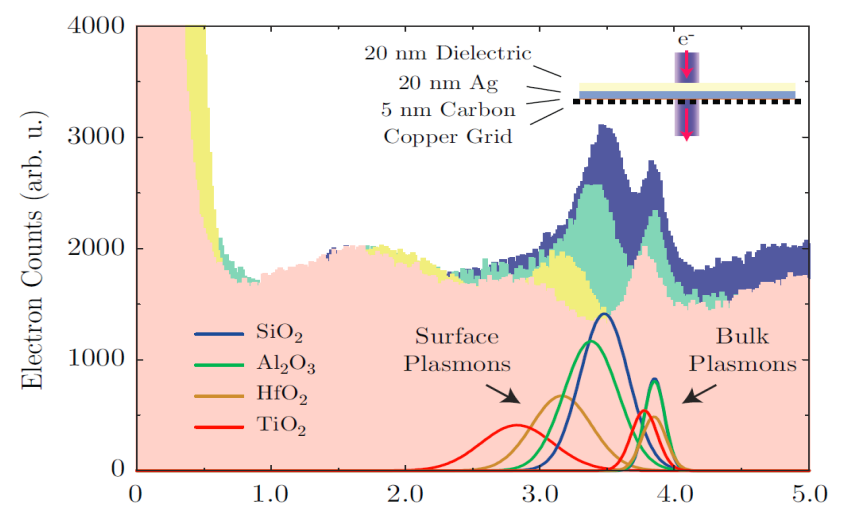

Figure 2. Monochromated electron energy-loss spectra. The colored column plots display the electron counts. The colored curves show the peaks via the standard multi-Lorentzian fitting.
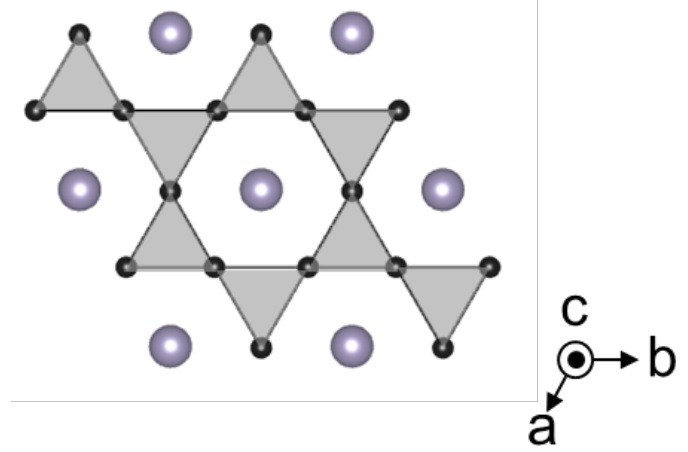

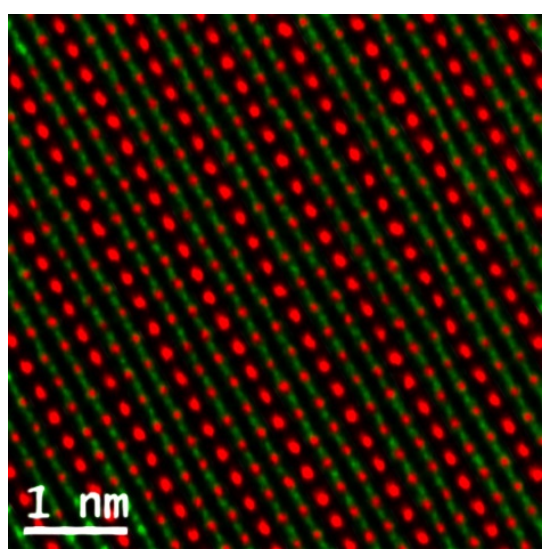

Figure 3. Spin-Obit Kagome Metal, $\mathrm{Fe}_{3} \mathrm{Sn}_{2}$. Structure (left) and LV-EELS Map (gaussian smoothed imaged) (right). 